\title{
Analysis of Cytotoxic Effects of Chemotherapeutic Agents for Gastrointestinal Cancer with Cell-based Impedance Biosensor
}

\author{
Senbiao Luo, ${ }^{*}$ Longshan Zhou, ${ }^{1}$ Yefeng Dai, ${ }^{1}$ Yanli Lu, ${ }^{2}$ and Qingjun Liu ${ }^{2}$ \\ ${ }^{1}$ Shangyu People's Hospital of Zhejiang Province, Shaoxing 312300, P. R. China \\ ${ }^{2}$ Department of Biomedical Engineering, Zhejiang University, Hangzhou 310027, P. R. China
}

(Received January 15, 2018; accepted June 5, 2018)

Keywords: cell-based impedance biosensor, gastrointestinal cancer, cytotoxic effects, chemotherapeutic agents

As one of the major causes of cancer-related death, gastrointestinal cancer seriously impacts the quality of human life. Moreover, in the majority of patients, the disease is in an advanced state at the time of diagnosis. The only available treatment for such patients is systemic chemotherapy, which may cause various side effects. Thus, the need for fast evaluation functions of different medicines and developing new drugs have become urgent in recent years. In this study, four different anti-cancer reagents, namely, compound Kushen injection (CKI), cinobufacini injection, transforming growth factor- $\beta 1$ (TGF- $\beta 1$ ), and 5-fluorouracil (5FU), were each tested with a cell-based biosensor to investigate their therapeutic effects on gastrointestinal cancer cells. From the impedance results, we could infer that chemotherapeutic agents could induce cell apoptosis, disrupt cell cycle, and inhibit cell adhesion capacity. By combining impedance spectra with an appropriate impedance circuit, the function of 5-FU on cell death and adhesion could be preliminarily investigated. Because the impedance sensing is in real time, has high throughput, and is easily manipulated, the developed cell-based impedance biosensor could be used as a suitable and promising alternative to end-point assay in the preclinical research of medicines, including the development of new therapeutic regimens.

\section{Introduction}

Gastrointestinal cancer is one of the most commonly diagnosed cancers, and one of the highest causes of cancer death for both men and women. ${ }^{(1-4)}$ It was reported that there were more than 49000 deaths due to gastrointestinal cancer in the United States in 2016. ${ }^{(5,6)}$ Moreover, in the majority of these patients, the disease was in an advanced state at the time of diagnosis. These sufferers had few opportunities for surgery, because the success rate of surgical treatment is very low. The only available treatment for them was systemic chemotherapy. ${ }^{(4)}$ Although one of the most important treatment methods clinically, chemotherapy has serious side effects. For gastrointestinal cancer, the most commonly used drugs are 5-fluorouracil (5-FU) and oxaliplatin (L-OHP), which could inhibit cell proliferation,

*Corresponding author: e-mail: passerer@aliyun.com

https://dx.doi.org/10.18494/SAM.2018.1910 
block the cell cycle, and interrupt DNA replication. ${ }^{(7-10)}$ However, 5-FU often harms the kidneys and digestive system, while L-OHP may cause hematologic toxicity and gastrointestinal tract toxicity. ${ }^{(11-13)}$ It is urgent to investigate new effective drugs to improve the life quality of patients.

In addition to chemical medicines, other kinds of drugs have also been investigated in recent years, such as some Chinese traditional medicines and biologics, including compound Kushen injection (CKI), cinobufacini injection, and growth factors. ${ }^{(14-17)}$ These Chinese traditional medicines could inhibit protein synthesis in cancer cells through DNA and RNA routes, and ultimately induce the apoptosis of cancer cells. Moreover, clinical studies showed that both CKI and cinobufacini injection could attenuate the side effects of chemotherapy and radiotherapy, thus improving the quality of life. ${ }^{(18,19)}$ Growth factors, such as transforming growth factors- $\beta$ (TGF- $\beta$ s) and fibroblast growth factors (FGFs), are secreted polypeptides and are involved in a variety of important cellular functions, including cell proliferation, differentiation, and apoptosis. $^{(15,20,21)}$ Therefore, detecting the impacts of these reagents on different cancer cells could provide a new way to explore the mechanism of cancer therapy.

Because of the extensive experimental process and stringent laboratory conditions, conventional methods, such as fluorescent imaging, radioactive detection, and animal experiments, do not meet the demands of assessing the efficacy of different medicines rapidly and continuously. In contrast to these end-point assays, biosensors with whole living cells have proven to be powerful and efficient tools for the real-time and label-free monitoring of the weak cell responses to drugs or other environmental stimuli. To achieve sufficient detection for interpreting the early effects of drugs on cells for cancer therapy or anti-cancer drug screening, various strategies have been explored. ${ }^{(22-26)}$ Among them, biosensors based on the electric cellsubstrate impedance sensing (ECIS) technique are promising for monitoring cell proliferation, morphology, motility, and apoptosis induced by different medicines. They can rapidly quantify the toxicity and selectivity of potential medicines to targeted cells, and could be used as a potential alternative to end-point assay in preclinical research, the development of new drugs, and toxicological testing.

In this study, using a cell-based impedance biosensor, the cytotoxic effects of four different chemotherapeutic agents, namely, 5-FU, CKI, cinobufacini injection, and TGF- $\beta 1$, on gastrointestinal cancer cells were detected and analyzed. Through the detection of the timeand concentration-dependent impedance changes of cancer cells on interdigitated electrodes exposed to different drugs, the condition of the cells could be evaluated, and may also be used to continuously monitor cell viability and evaluate the functions of anti-cancer drugs. By revised impedance circuit modeling, the impedance changes of the cell-based biosensor were extracted and analyzed under stimulation by 5-FU. By directly showing the state of gastrointestinal cancer cells treated with different medicines, this research is significant for the in vitro exploration of the anti-cancer mechanism of medicines, especially Chinese traditional medicines and biologics. 


\section{Materials and Methods}

\subsection{Reagents}

The following reagents were used: 5-FU injection (Tianjin Jinyao Amino Acid Co., Ltd., China), CKI (Shanxi Zhendong Pharmaceutical Co., Ltd., China), cinobufacini injection (Anhui Jinchan Biochemistry Corporation, China), and TGF- $\beta 1$ (Abcam, Cambridge, MA). All of these reagents were diluted with culture medium into different concentrations. Cells in a negative control group were treated with culture medium.

\subsection{Impedance sensor and cell culture}

The sensor comprising an electronic plate (E-Plate 16) with interdigitated electrodes at the bottom of 16 wells was obtained from ACEA Biosciences (USA) [Fig. 1(a)]. Each well had a volume of $243 \pm 5 \mu \mathrm{L}$ to culture cells on electrodes. The interdigitated electrodes at the bottom of the wells had the shape of a circle on a line [Fig. 1(b)]. The interdigitated electrodes covered approximately $80 \%$ of the bottom area of each well, providing maximal sensitivity for the detection of the electrochemical reactions at the electrode interface and relatively uniform distribution of the electric field.

Colorectal cancer cells (LS180, SW620) (American Tissue Culture Collection, CCL131) were maintained in Roswell Park Memorial Institute (RPMI)-1640 medium supplemented with $10 \%$ fetal calf serum (FCS; heat-inactivated), 100 units $/ \mathrm{mL}$ penicillin $\mathrm{G}$, and $100 \mu \mathrm{g} / \mathrm{mL}$ streptomycin. All cells were cultured at $37{ }^{\circ} \mathrm{C}$ in a humidified atmosphere containing $5 \% \mathrm{CO}_{2}$. Cancer cells were planted in the microchamber wells of the sensor device at a density of $1 \times 10^{5}$ cells $/ \mathrm{mL}$ monitored by a standard hemacytometer. After seeding, the cells underwent a time-

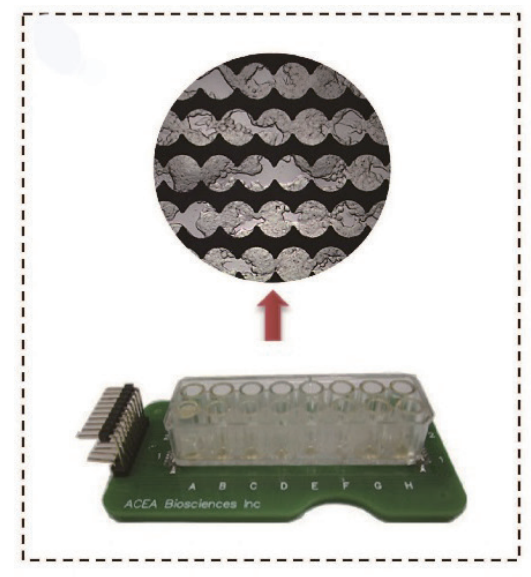

(a)

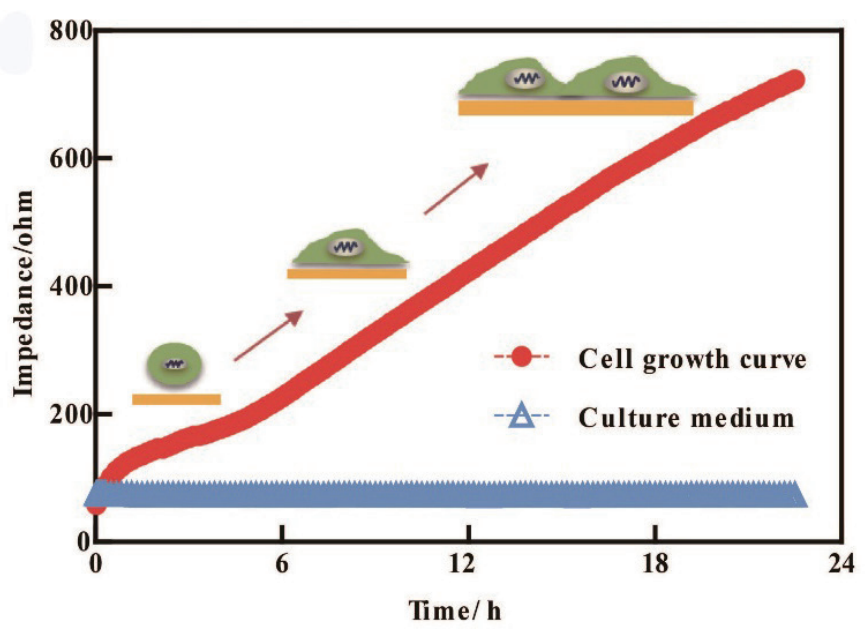

(b)

Fig. 1. (Color online) Impedance sensor and cell culture. (a) E-Plate 16 device of cell-based impedance biosensors with interdigitated electrodes cultured with cancer cells. (b) Schematic of cell deposition, growth, and proliferation on the electrodes with the detected cell growth curve. 
dependent morphology change, and $24 \mathrm{~h}$ later, they were spread over the electrode [Fig. 1(a)]. System impedance was measured on the Zahner ZENNIUM electrochemical workstation (Zahner Elektrik, Germany) with tested frequency from $1 \mathrm{kHz}$ to $1 \mathrm{MHz}$. The real-time impedance response of $10 \mathrm{kHz}$ to cell deposition, growth, and proliferation on the electrode surface is shown in Fig. 1(b). All the measurements were performed at $37 \pm 0.2{ }^{\circ} \mathrm{C}$ in an incubator.

\subsection{Cell index detection}

Before cell seeding, a background resistance was recorded by adding $240 \mu \mathrm{L}$ of cell culture medium. Then, an equal volume of cell suspension was added into different wells, and the recording of cell index $(C I)$ at a fixed frequency $(10 \mathrm{kHz})$ every 10 min was started to obtain real-time recordings using an iCELLigence system (ACEA Biosciences, USA).

$$
C I=\frac{Z_{c e l l}-Z_{b}}{Z_{b}}
$$

Here, $Z_{c e l l}$ represents the resistance of the system in the presence of cells, and $Z_{b}$ represents the resistance of the system before cell seeding.

After $24 \mathrm{~h}$, the culture medium in each plate well was refreshed. Cell culture medium and culture solution containing different anti-cancer drugs were injected alternately. In the control experiments, the cancer cells were treated with culture medium. The cell index was recorded for another $24 \mathrm{~h}$. The toxicity reflected by the cell index was the gross cytotoxicity of different drugs of different concentrations, which had effects on the cell biological status including cell viability, cell number, morphology, and adhesion. ${ }^{(26-28)}$

\section{Results and Discussion}

\subsection{Impedance circuit modeling}

During the electrochemical measurement, an alternating voltage $(20 \mathrm{mV})$ was applied. Generally, culture medium on electrodes is equivalent to a simple circuit containing a solution resistance $\left(R_{S}\right)$ and a constant phase element (CPE) [Fig. 2(a)]. When the gastrointestinal cancer cell suspension solution was added to each plate well, round cells would gradually attach and spread over the electrodes. On the basis of the pioneer work of Giaever and Keese, ${ }^{(29,30)}$ various impedance models of cells were simplified to be a resistor-capacitor (RC) parallel circuit at last. It is one of the most common impedance circuits used to characterize the properties of cells. Besides $R_{S}$ and CPE, the impedance in the presence and absence of the cells is distinguished by the cell impedance, which is composed of $R_{c}$ and $C_{c}$ [Fig. 2(b)]. Finally, cells reached a state with the largest contact area [Fig. 2(c)], and electric current could only flow through the cell interval, which lead to another resistance $R_{b}$ that can be used to evaluate the tightness of the attachment between cells and electrodes. Thus, the revised electrical circuit was used to discuss the cell adhesion, spreading, and micromotion referring to the results of simulations using Zveiw (Scribner Associates Inc., US). The detailed parameters of the circuit could be used to 

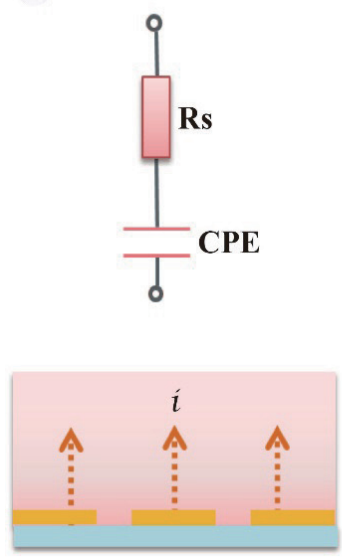

(a)
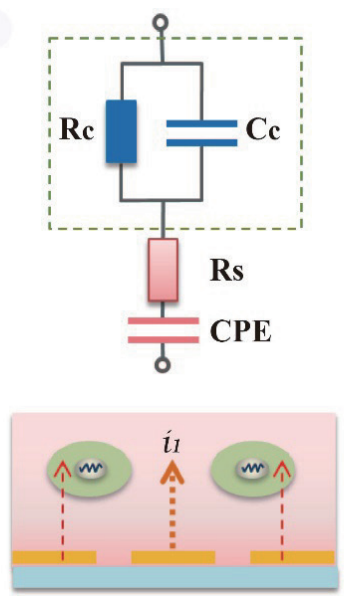

(b)
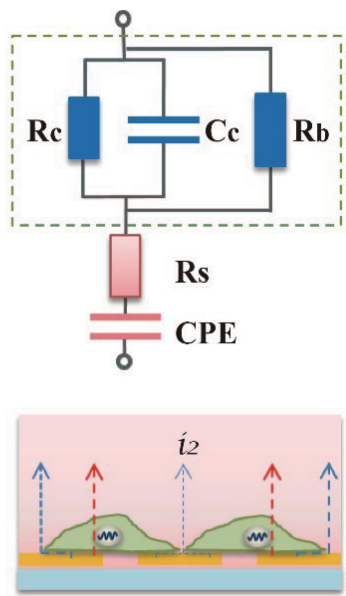

(c)

Fig. 2. (Color online) Cell morphology and equivalent circuits on electrodes. (a) Culture medium on electrodes with equivalent circuit. (b) Cell morphology and its equivalent circuit when cells are suspended in medium. (c) Cell morphology and its equivalent circuit when cells are attached to electrodes. $R_{S}$ refers to the resistance of the culture medium, CPE, $R_{c}$, and $C_{c}$ are the resistance and capacitance of cells, respectively, $R_{b}$ is the resistance both between adjacent cells and between cells and electrodes.

explain changes in the cell conditions, including those of the membrane and nucleus, owing to anti-cancer drugs at the same time.

As a powerful method for the characterization of the structural features of the sensing interface, impedance spectra can be used to explain the mechanism of biochemical processes occurring at the electrode-solution interfaces. ${ }^{(26,31)}$ Examination of the impedance changes enables the analysis of interfacial changes originating from biorecognition events or cell adhesion at electrode surfaces and provides real-time and kinetic information of cell behaviors. Thus, the cell-based impedance biosensors could be used to monitor the conditions of cancer cell attachment, cell proliferation, and cell detachment by exposing cells to different medicinal compounds.

\subsection{Impedance detection and analysis of Chinese medicines}

Generally, cell impedance was affected by multiple parameters, including cell number, adhesion, and morphology, all of which can be influenced by cytotoxic compounds. ${ }^{(27)}$ Through real-time monitoring, cell index was found to reflect the overall condition of cancer cells. During the first $24 \mathrm{~h}$, cancer cell proliferation, attachment, and spreading on the electrodes resulted in impedance increases (Fig. 3). However, when cancer cells in plate wells were treated with different medicines at different concentrations, the cell index showed various changes depending on the mechanism of the compound action. Over the lengthy course of toxicant exposure, the attached and infected cells eventually die and/or cannot bind firmly to the gold electrodes, corresponding to a gradual decrease in cell index. 


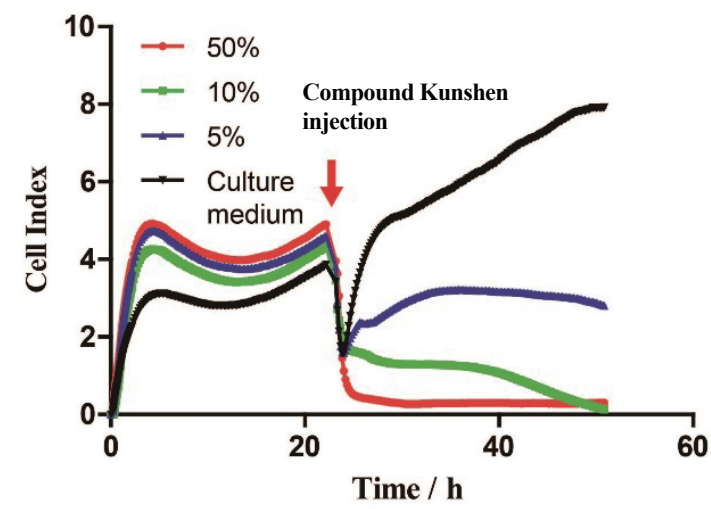

(a)

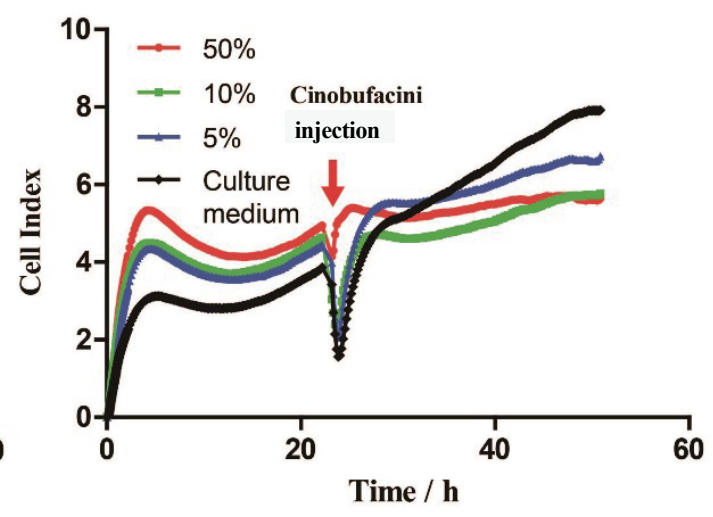

(b)

Fig. 3. (Color online) Cell index with CKI (a) and cinobufacini injection (b); the legend shows the volume ratio of medicine in culture medium.

Compared with the control group without any medicine, both CKI and cinobufacini injection had considerable effects on colon cancer cells. We could also infer that with higher concentrations of these two drugs, the more the impedance changed. Additionally, changes in the cell impedance depended on how much of the drugs the cells were exposed to, and for how long. With CKI treatment, however, the decrease in the cell index was very different from that in the case of cinobufacini injection, which indicated that these two traditional Chinese medicines have distinct mechanisms and curative effects.

As shown in Fig. 3(a), the sharp decline in the CKI cell index at the volume ratio of 50\% might be caused by multiple factors, such as a decrease in cell number, reduction in spreading area, and attenuation of adhesive forces. The results indicated that CKI could destroy the cytoskeleton, and even induce cell apoptosis. The impedance changes also appeared in a timedependent pattern, especially at the drug concentration of $10 \%$. With longer action time of the drug, CKI could also cause cancer cell apoptosis. However, when the drug concentration was $5 \%$, colon cancer cells would still be alive after $24 \mathrm{~h}$, which suggested that the main function of CKI at low concentrations is as a cytostatic.

CKI is extracted from the root of two herbs, Sophora flavescens and Heterosmilacis japonica. It had been extensively used alone or in combination with chemotherapy or radiotherapy in Chinese clinical settings for many years. ${ }^{(18,32)}$ The primary components of the medicine are matrine and oxymatrine that suppress tumor cell growth by inducing apoptosis and inhibiting adhesion. The changes in the cell index indicated that CKI has a significant antitumor activity and that impedance sensing is a promising method for exploring the CKI function.

The injection of cinobufacini, an aqueous compound extracted from the skin of the Asiatic toad Bufo bufo gargarizans Cantor, is also widely used in clinical cancer therapy in China. ${ }^{33,34)}$ Different from CKI, upon cinobufacini injection treatment, the cancer cell index did not decrease, but remained in a relatively steady state during measurements [Fig. 3(b)]. Compared with the control group, the cell index of cancer cells slightly increased after injecting cinobufacini. More importantly, only small differences were observed between the impedance spectra after cinobufacini injection at different concentrations, 50, 10, and 5\%. The results 
suggested that the effect of cinobufacini on human SW620 cells is predominantly disruption of the cell cycle and suppression of cell division, with little influence on the cytoskeleton. This is in agreement with previous research, which indicated that cinobufacini affects the cell surface, induces cell cycle arrest at the $\mathrm{S}$ phase, and inhibits cellular proliferation significantly. ${ }^{(35)}$

Taken together, the above results suggested that CKI induces the apoptosis of colon cancer cells, whereas cinobufacini mainly inhibits cellular proliferation. By monitoring the impedance changes of colon cancer cells, the cytotoxic effects of the two kinds of traditional Chinese anticancer drugs were analyzed. The results provided experimental evidence of the efficacy of CKI and cinobufacini in the treatment of colon cancer cells. Compared with conventional methods used in cancer cell biology, including methyl thiazolyl tetrazolium (MTT), radioactive detection, fluorescent imaging, and even animal experiments, ${ }^{(36-38)}$ the cell-based biosensor offers a realtime, inexpensive, and easy method of continuously monitoring cell conditions.

\subsection{Impedance sensing of growth factor}

Because of the control of a wide range of biological functions, regulation of cellular proliferation, survival, migration, and differentiation through signaling, various growth factors were treated as important cancer therapeutic targets, such as TGF- $\beta$ s and FGFs. ${ }^{(15,20,21)}$ Here, we take TGF- $\beta 1$ as an example to investigate the effect of TGF- $\beta$ s on colon cancer cells. TGF- $\beta 1$ solution was prepared at 6 different concentrations with culture medium.

As shown in Fig. 4, there was a gradual increase in the normalized cell index when only culture medium was applied. For low concentrations, $0.01,0.025$, and $0.05 \mu \mathrm{g} / \mathrm{mL}$, of TGF- $\beta 1$, the normalized cell index also increased, but at a much lower rate than that of the control group (Fig. 4). Meanwhile, the ability to inhibit the growth of colon cancer cells is shown in concentrationand time-dependent manners. For high concentrations, 0.5 and $0.25 \mu \mathrm{g} / \mathrm{mL}$, however, the normalized cell index increased substantially, which indicated that TGF- $\beta 1$ at high concentration could prompt cell growth effectively. When the concentration of TGF- $\beta 1$ was about $0.1 \mu \mathrm{g} / \mathrm{mL}$, changes in the cell index were almost the same as that with culture medium. From the results, we could infer that TGF- $\beta 1$ could trigger a variety of different cellular responses depending on its concentration.

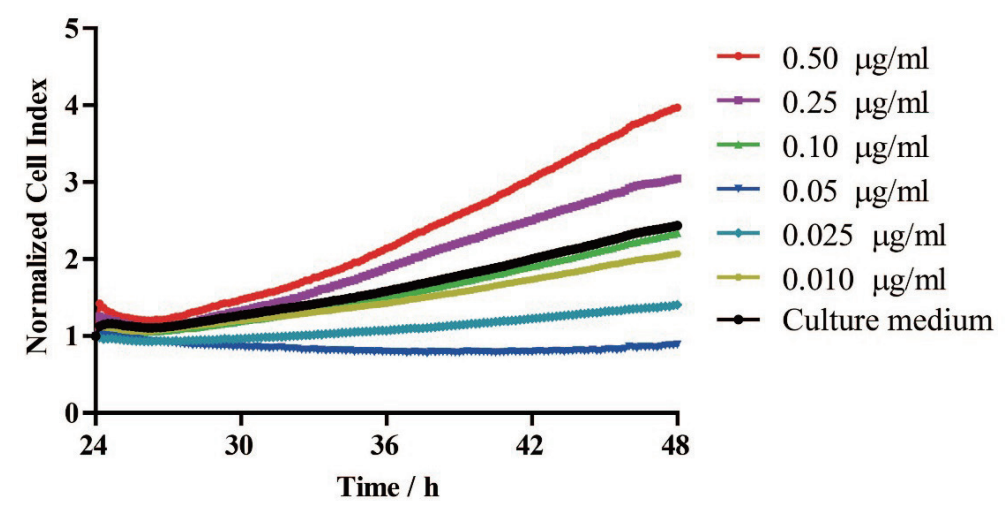

Fig. 4. (Color online) Normalized cell index of cancer cells with TGF- $\beta 1$ at different concentrations. 
TGF- $\beta$ s are multifunctional cytokines important in diverse biological processes, including development, differentiation, and immunoregulation. Their signaling is one of the key pathways altered in inflammatory bowel disease-associated colorectal cancer. ${ }^{(39)}$ For TGF- $\beta 1$ in this study, it was essential to maintain homeostasis between cell growth and apoptosis. Indeed, it played dual roles in cancer cell proliferation, and the provided signals for cell survival or apoptosis were dependent on cell type and condition. ${ }^{(20,40,41)}$ The results obtained with the cell-based biosensor indicated that the effects TGF- $\beta 1$ on colon cancer cells were dependent on concentration. It was in accordance with the fact that all human tumors overproduce TGF- $\beta 1$, whose autocrine and paracrine actions promote tumor cell invasiveness and metastasis. ${ }^{(20)}$ Therefore, impedance sensing offers an easy way to evaluate the function of the TGF- $\beta$ signaling cascade in gastrointestinal carcinogenesis and provides some new molecular insights that may aid efforts towards targeted antitumor therapies.

\subsection{Impedance model analysis}

5-FU has been the mainstay in gastrointestinal cancer treatment over the last 40 years. It is converted to three major active metabolites: (1) fluorodeoxyuridine monophosphate (FdUMP), (2) fluorodeoxyuridine triphosphate (FdUTP), and (3) fluorouridine triphosphate (FUTP). FUTP can disrupt RNA synthesis. FdUMP can inhibit the action of thymidylate synthase (TS), a nucleotide synthetic enzyme, and FdUTP can be directly misincorporated into DNA. Hence, the therapeutic target of 5-FU is the cell nucleus. ${ }^{(27)}$

In our study, impedance changes of 5-FU had their own characteristics, from which we could also infer that the mechanism of action is at the cell nucleus. The changes in the normalized cell index of 5-FU depend on its concentration. Higher concentrations correspond to a larger impedance change (Fig. 5). Additionally, after a short time of increase, the impedance decreased slowly, indicating cell apoptosis. However, from the cell index, it was difficult to distinguish whether the main factor was cell death or detachment.

Here, an equivalent electrical circuit [shown in Fig. 2(c)] was used to investigate the responses of the cancer cells under treatment with medicine. Taking the impedance data of

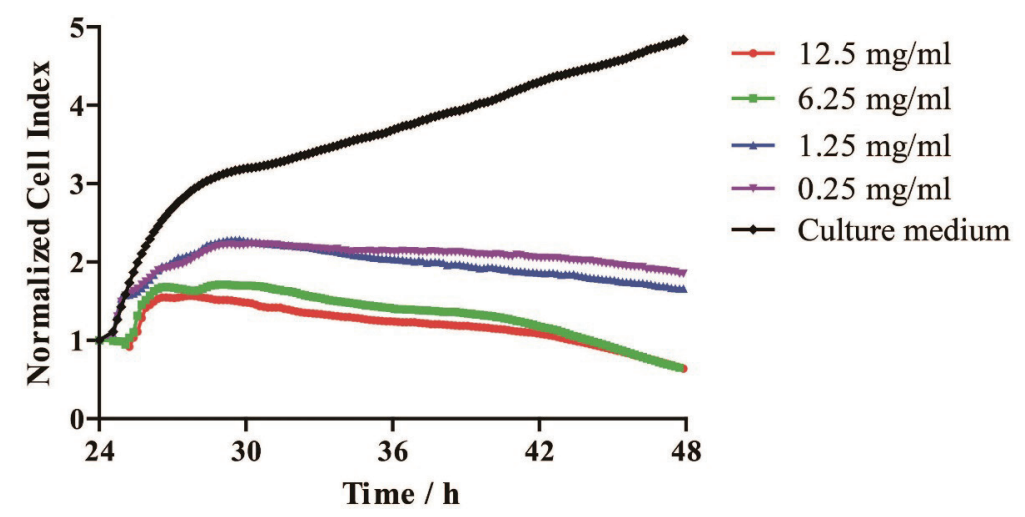

Fig. 5. (Color online) Cell index changes in cancer cells with 5-FU at different concentrations. 
5-FU at $12.5 \mathrm{mg} / \mathrm{mL}$ as an example, we regarded the characteristics in analogy with the curves of the circuit and summarized the fitted parameters of some important elements in Table 1, to investigate the cytotoxicities of drugs.

In the fitted results, the decrease in $R_{b}, 15 \%$, indicated that under treatment with 5-FU, cells detached from the electrodes. Additionally, changes in $C_{c}$ and $R_{c}$ represented the overall characteristics of cancer cells. The capacitance of cells decreased by $68 \%$, indicating a considerable reduction in the lipid bilayer capacitance, which was mainly influenced by the capacitances of the cell and the nuclear membrane. Compared with $C_{c}$, the resistance of cells $\left(R_{c}\right)$ increased little, about 39\%. Actually, cell death caused a reduction in $R_{c}$, while cell detachment from electrodes led to a larger resistance. Therefore, cell detachment played an important role in the increase in cell resistance. On the other hand, 5-FU can stop the cell proliferation by blocking the cell cycle after DNA replication, which leads to increasing resistance of the cell nucleus. Through the analysis of these detailed parameters of the impedance model, therapeutic targets and the efficiency of 5-FU could be investigated preliminarily. Therefore, by combining the fitted results of the model with the changes in the cell membrane and nucleus under treatment, this circuit model could be extended to the analysis of other medicines in the future.

The anti-cancer reagents CKI, cinobufacini injection, TGF- $\beta 1$, and 5-FU have different therapeutic targets, which could cause various cytotoxic effects on colon cancer cells. From the impedance spectra of the cell-based biosensor, diverse changes in cancer cells could be identified. The sharp decline in the impedance spectra with CKI indicated that CKI could suppress cell growth by inducing apoptosis and inhibit the adhesion capacity. The gentle changes in the impedance spectra with cinobufacini injection suggested that it could only disrupt the cell cycle and suppress cell division. TGF- $\beta 1$ played dual roles in cancer cell proliferation depending on its concentration. In contrast, 5-FU affects the cell nucleus and could suppress cell growth. By combining this cell-based biosensor with impedance model analysis, a promising method of function mechanism investigation in new medicine research was explored. It may possibly promote the development of diagnostics and therapies for colon cancer.

Compared with traditional viability assays (end-point viability readout), such as the WST1 , the cell-based biosensor, which is simple, sensitive, and low in cost, could be applied for studying the real-time and kinetic information of cell behavior, such as cell proliferation, morphology, and motility. ${ }^{(26,42)}$ The ability of rapidly quantifying the toxicity of potential anti-cancer medicines to targeted cancer cells would provide an efficient method for the rapid screening of drugs in preclinical research.

Table 1

Impedance fitted results with parameters of cells and cell adhesion.

\begin{tabular}{lccc}
\hline & $C_{c} / F$ & $R_{c} / \Omega$ & $R_{b} / \Omega$ \\
\hline Before & $1.9 \times 10^{-7}$ & $6.1 \times 10^{2}$ & $1.3 \times 10^{2}$ \\
After & $6.1 \times 10^{-8}$ & $8.5 \times 10^{2}$ & $1.1 \times 10^{2}$ \\
Changes & Decrease by $68 \%$ & Increase by $39 \%$ & Decrease by $15 \%$ \\
\hline
\end{tabular}




\section{Conclusion}

With the cell-based impedance biosensor, the cytotoxic effects of different reagents, namely, CKI, cinobufacini injection, TGF- $\beta 1$, and 5-FU, on gastrointestinal cancer cells could be evaluated. Different drugs at various concentrations caused diverse pharmacodynamics in colon cancer cells, which could be inferred from the patterns of impedance changes. By combining impedance spectra with appropriate impedance circuit modeling, we could preliminarily estimate the effects of different medicines on targeted cancer cells. Because the impedance sensing is in real time, has high throughput, and is easily manipulated, this biosensor could be used as a suitable and promising alternative to end-point assay in the preclinical research of medicines, including the development of new therapeutic regimens.

\section{Acknowledgments}

This work was supported by the Research on Public Welfare Technology Application Projects of Zhejiang Province (Grant No. 2014C33149).

\section{References}

1 A. Goel and C. R. Boland: Curr. Opin. Gastroenterol. 26 (2010) 47.

2 A. Tenesa and M. G. Dunlop: Nat. Rev. Genet. 10 (2009) 353.

3 R. L. Siegel, K. D. Miller, S. A. Fedewa, D. J. Ahnen, R. G. Meester, A. Barzi, and A. Jemal: CA-Cancer J. Clin. 67 (2017) 177.

4 B. Gustavsson, G. Carlsson, D. Machover, N. Petrelli, A. Roth, H.-J. Schmoll, K.-M. Tveit, and F. Gibson: Clin. Colorectal Cancer 14 (2015) 1.

5 R. L. Siegel, K. D. Miller, and A. Jemal: CA-Cancer J. Clin. 66 (2016) 7.

6 R. A. T. Stone, M. E. Waring, S. L. Cutrona, C. I. Kiefe, J. Allison, and C. A. Doubeni: BMJ Open Sci. 7 (2017) e015619.

7 S. Giacchetti, M. Itzhaki, G. Gruia, R. Adam, R. Zidani, F. Kunstlinger, S. Brienza, E. Alafaci, F. BertheaultCvitkovic, and C. Jasmin: Ann. Oncol. 10 (1999) 663.

8 L. R. Wiseman, J. C. Adkins, G. L. Plosker, and K. L. Goa: Drug Aging 14 (1999) 459.

9 C. V. De Almeida, J. A. Zamame, G. G. Romagnoli, C. P. Rodrigues, M. B. Magalhães, A. Amedei, and R. Kaneno: Oncol. Rep. 38 (2017) 561.

10 S. Toden, Y. Okugawa, T. Jascur, D. Wodarz, N. L. Komarova, C. Buhrmann, M. Shakibaei, C. R. Boland, and A. Goel: Carcinogenesis 36 (2015) 355.

11 T. Esaki, S. Nakano, T. Tatsumoto, M. Kuroki-Migita, K. Mitsugi, M. Nakamura, and Y. Niho: Cancer Res. 52 (1992) 6501.

12 J. Kjellström, E. Kjellén, and A. Johnsson: Acta Oncol. 44 (2005) 687.

13 H. Weh, H. Wilke, J. Dierlamm, U. Klaassen, R. Siegmund, H. Illiger, A. Schalhorn, E. Kreuser, U. Hilgenfeld, and B. Steinke: Ann. Oncol. 5 (1994) 233.

14 B. Yanju, L. Yang, B. Hua, W. Hou, Z. Shi, W. Li, C. Li, C. Chen, R. Liu, and Y. Qin: Support. Care Cancer 22 (2014) 825.

15 N. Turner and R. Grose: Nat. Rev. Cancer 10 (2010) 116.

16 Z. Qu, J. Cui, Y. Harata-Lee, T. N. Aung, Q. Feng, J. M. Raison, R. D. Kortschak, and D. L. Adelson: Oncotarget 7 (2016) 66003.

17 D. Chen, R. Zhang, Y. Liu, T. Zhou, X. Li, S. Gao, J. Zhang, and X. Cui: RSC Adv. 6 (2016) 82417.

18 W. Xu, H. Lin, Y. Zhang, X. Chen, B. Hua, W. Hou, X. Qi, Y. Pei, X. Zhu, and Z. Zhao: J. Exp. Clin. Cancer Res. 30 (2011) 103.

19 F. Qi, A. Li, Y. Inagaki, N. Kokudo, S. Tamura, M. Nakata, and W. Tang: Int. Immunopharmacol. 11 (2011) 342.

20 K. Pardali and A. Moustakas: Biochim. Biophys. Acta, Biomembr. 1775 (2007) 21. 
21 P. Lampropoulos, A. Zizi-Sermpetzoglou, S. Rizos, A. Kostakis, N. Nikiteas, and A. G. Papavassiliou: Cancer Lett. 314 (2012) 1.

22 A. Pallaoro, M. R. Hoonejani, G. B. Braun, C. D. Meinhart, and M. Moskovits: ACS Nano 9 (2015) 4328.

23 Y. Wang, S. Zhang, T. Xu, T. Zhang, Y. Mo, J. Liu, L. Yan, and F. Xing: Sens. Actuators, B 263 (2018) 417.

24 M. Dervisevic, M. Senel, T. Sagir, and S. Isik: Biosens. Bioelectron. 90 (2017) 6.

25 H. Abiri, M. Abdolahad, M. Gharooni, S. A. Hosseini, M. Janmaleki, S. Azimi, M. Hosseini, and S. Mohajerzadeh: Biosens. Bioelectron. 68 (2015) 577.

26 Q. Liu, Y. Lu, H. Wang, J. Zhou, Y. Zhang, Q. Chen, S. Luo, R. Li, and P. Wang: Anal. Lett. 47 (2014) 1348.

27 J. M. Atienza, N. Yu, S. L. Kirstein, B. Xi, X. Wang, X. Xu, and Y. A. Abassi: Assay Drug Dev. Technol. 4 (2006) 597.

28 L. Hu, L. Zou, Z. Qin, J. Fang, L. Huang, and P. Wang: Sens. Actuators, B 238 (2017) 1151.

29 H.-H. Huang: Biochem. Biophys. Res. Commun. 314 (2004) 787.

30 S. Cho and H. Thielecke: Microelectron. Eng. 85 (2008) 1272.

31 Q. Liu, C. Wu, H. Cai, N. Hu, J. Zhou, and P. Wang: Chem. Rev. 114 (2014) 6423.

32 Z. Zhao, H. Fan, T. Higgins, J. Qi, D. Haines, A. Trivett, J. J. Oppenheim, H. Wei, J. Li, and H. Lin: Cancer Lett. 355 (2014) 232.

33 F. Qi, A. Li, Y. Inagaki, H. Xu, D. Wang, X. Cui, L. Zhang, N. Kokudo, G. Du, and W. Tang: Food Chem. Toxicol. 50 (2012) 295.

34 R.-F. Xie, Z.-C. Li, B. Gao, Z.-N. Shi, and X. Zhou: J. Ethnopharmacol. 141 (2012) 692.

35 L. Ma, B. Song, H. Jin, J. Pi, L. Liu, J. Jiang, and J. Cai: Bioorg. Med. Chem. Lett. 22 (2012) 1459.

36 T. Mosmann: J. Immunol. Meth. 65 (1983) 55.

37 Y. Liu, D. A. Peterson, H. Kimura, and D. Schubert: J. Neurochem. 69 (1997) 581.

38 F. Chen, J. Tu, C. Liang, B. Yang, C. Chen, X. Chen, and C. Cai: Microchim. Acta 183 (2016) 1571.

39 S. G. Daniel, C. L. Ball, D. G. Besselsen, T. Doetschman, and B. L. Hurwitz: mSystems 2 (2017) e00065.

40 R. Yi, Y. Li, F. Wang, J. Gu, T. Isaji, J. Li, R. Qi, X. Zhu, and Y. Zhao: Tumor Biol. 37 (2016) 10763.

41 W. Wen, G. Liu, K. Jin, and X. Hu: Oncol. Rep. 37 (2017) 115.

42 C. R. Keese, J. Wegener, S. R. Walker, and I. Giaever: Proc. Natl. Acad. Sci. U.S.A. 101 (2004) 1554. 